\title{
Medicine Cabinet
}

\section{Vaccination updates}

\section{INTRODUCTION}

New developments have occurred in vaccination, leading to changes in existing vaccines and vaccination policies in children and adults. The following information is based on reports that appeared in Drug Therapy Topics of the University of Washington Medical Center, Seattle.

\section{UPDATES IN PEDIATRIC VACCINATION Chickenpox vaccine}

The Food and Drug Administration approved the nation's first chickenpox vaccine in 1995. The Centers for Disease Control and Prevention (CDC) and the American Academy of Pediatrics now recommend immunizing children against the infection at 12 to 18 months of age. Approval followed rancorous debate about the need for universal vaccination against a self-limiting disease. Since its approval, many parents have expressed reluctance about giving their child yet another vaccine. A recent parental survey, however, suggested that parents feel positive about the vaccine's ability to prevent the rare but serious complications of chickenpox. $^{1}$

The most persuasive evidence to date of the efficacy of chickenpox vaccination comes from a field study conducted by the CDC in an area north of Los Angeles. California has the highest chickenpox rates in the country. Five years after launching a voluntary chickenpox vaccination program, health officials found that inoculations can reduce cases by $80 \%{ }^{2}$ The number of chickenpox cases in the region fell from 2,934 in 1995 to 587 by 1999. Rates declined in all age groups. The most impressive decline occurred in children ages 1 through 4 years. The vaccine also showed economic and social benefits. Infected children and their caretakers in the region missed 14,842 days of work and school in 1995 but only 2,718 days in 1999.

Based on these results, California will make chickenpox vaccination mandatory in 2001 for children entering its schools or day-care facilities. Half the states in the country now require the vaccination.

\section{Combination vaccine use}

A meeting at the National Institutes of Health concluded that combination vaccines, containing several antigenic components, are essential if physicians are to take full advantage of vaccines under development to treat childhood disease.

The number of injections now included or to be added to the pediatric immunization schedule is imposing an unacceptable burden on physicians and parents. The current schedule involves giving a child 19 to 23 injections by 6 years of age, 16 of them in the first 18 months. One way

\section{Summary points}

- Vaccination against chickenpox can reduce chickenpox rates, and it will become mandatory in California in 2001

- Combination vaccines can minimize the burden of having to give multiple injections to children

- Healthy children younger than 2 years are at high risk of influenza and may benefit from influenza vaccination

- Vaccinating health care workers against influenza reduces the risk of death among elderly patients

- It is now recommended that everyone older than 50 years receive the influenza vaccine

to minimize this burden is to combine the necessary agents into fewer vaccines. At issue is how to test such combinations for safety and efficacy. ${ }^{3}$ Even the safety and efficacy of combination vaccines whose components are already licensed are not fully established.

\section{Young children may benefit from influenza vaccination}

Two independent studies have reported that healthy children younger than 2 years are hospitalized for influenza at higher rates than older children during the influenza season. The findings suggest that children in this age group are at high risk and should receive vaccination.

In 1 investigation, Neuzil and colleagues reviewed Medicaid files from 1973 to 1993 and identified healthy children up to age 15 years. ${ }^{4}$ They examined hospitalization rates for acute cardiopulmonary conditions, outpatient visits, and the use of antibiotics during and out of influenza season. The average number of excess hospitalizations was 4 per 10,000 children per year for 5- to 15 -year-olds. However, this number rose to 104 per 10,000 children per year for infants younger than 6 months. The excess number of outpatient visits was highest in those aged 6 to 12 months. Antibiotic use was far more prevalent in infants than in older children. The authors concluded, "The excess rate of hospitalization in [children younger than 1 year] is similar to rates in adults for whom an influenza virus vaccine is recommended." 4

In a second investigation, Izurieta and associates studied children aged 18 years or younger in several cities who were enrolled in health maintenance organizations from 1992 to 1997 . The research team examined hospitalization rates for acute respiratory disease during periods when the circulation of influenzaviruses predominated over that of respiratory syncytial virus. The hospitalization rate for low-risk children younger than 2 years was about 200 per
Milo Gibaldi

Department of Pharmaceutics University of Washington Medical Center

Box 357610, H272

Health Sciences Bldg

Seattle, WA 98195

Correspondence to: Dr Gibaldi gibaldi@u.washington. edu

Competing interests: None declared

West J Med 2000;173:269-271 
100,000 per month, similar to that for high-risk children aged 5 to 17 years. The hospitalization rate was more than 90\% lower in low-risk 5- to 17-year-olds. The authors concluded, "Our study demonstrates increased rates of influenza-related hospitalization among children younger than 2 years of age and suggests that routine influenza vaccination should be considered in these children."

An editorial accompanying these 2 papers cautions, "To justify routine influenza vaccination of infants and toddlers, the benefit-risk ratio would need to be very high." The authors are concerned by "the relatively small proportion of cases of severe respiratory illness that would be prevented, the probable need for more than one dose, the uncertainties and unpredictability of antigenic variability, and the need to immunize during a period when many other vaccines are given." They recommend that large controlled trials of infant and toddler influenza vaccination be done to evaluate safety, effectiveness, and costeffectiveness.

\section{UPDATES IN ADULT VACCINATION Vaccinating health care workers reduces influenza-related deaths in elderly patients}

Offering routine influenza vaccinations to health care workers in long-term care facilities has been shown to significantly reduce the risk of death among elderly patients. $^{7}$ In a randomized controlled trial that was carried out during a flu outbreak in the United Kingdom, the investigators offered or did not offer flu vaccine to the staff of 20 long-term care facilities. Only half of the health care workers who were offered flu vaccine were actually inoculated, compared with $5 \%$ of those who were not offered the vaccine. Nasal and throat swabs were taken for viral surveillance every 2 weeks from patients in these facilities during the outbreak. The researchers found that $13.6 \%$ of patients died in facilities where the staff was offered vaccine, which was significantly less than in the control facilities, where $22.4 \%$ died. The number of patients infected with influenza between the treatment and control facilities did not differ, however.

The findings suggest that a program of influenza vaccination of health care workers substantially lowers mortality among elderly patients, seemingly by reducing the severity of the infection. An accompanying editorial says that more studies are needed "before concrete steps are taken to routinely vaccinate health care workers."

\section{Vaccine modified for 2000-2001 influenza season}

The influenza vaccine is changed every year to reflect the strains that infectious disease experts predict will circulate. The flu vaccine licensed in the United States is made from killed influenzaviruses, and it cannot cause infection.

This year, the CDC's Advisory Committee on Immunization Practices has decided to replace 2 of the 3 strains

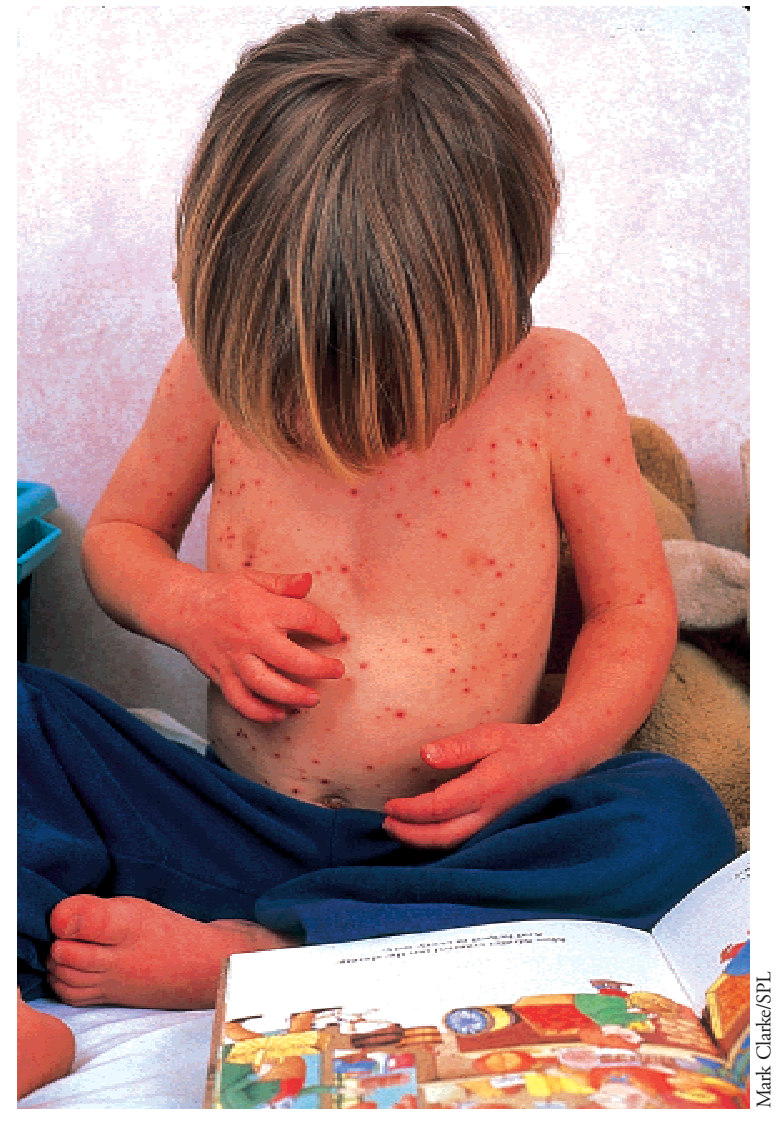

Chickenpox vaccination is now recommended for all children 12 to 18 months of age

in the 1999-2000 vaccine and to lower the age for which a flu shot is recommended. The expert committee has now recommended that the vaccine be given to people aged 50 years and older because almost a third of the adults in this age group has 1 or more medical conditions that put them at high risk of flu-related hospitalization and death. Previously, the vaccine was recommended for people aged 65 and older.

Most 1999-2000 cases of influenza infections were type $\mathrm{A}$, and lapses in prevention were reported. According to the CDC, failure to immunize results in 20,000 to 40,000 flu-related deaths each year in the United States.

\section{Manufacturing problems have delayed flu vaccine}

Officials at the CDC disclosed that the shipments of influenza vaccine for the $2000 \mathrm{flu}$ season will be delayed and that there may be shortages because of manufacturing problems. The agency told clinics, hospitals, and other providers of health care to delay mass immunization campaigns for at least a month and to prepare to immunize people at highest risk first. The chief of the influenza branch of the CDC described the delay as follows: "We have never had a situation where all manufacturers were having a similar problem with a strain." 
The announcement of the delay came at a time when health officials expected that a record number of Americans, perhaps as many as 75 million, would be immunized because of the lowering of the age to 50 years when all persons are advised to get immunization.

Drug Therapy Topics is a monthly publication from the University of Washington-Harborview Medical Center Drug Information Center. The full text is freely available at http://depts.washington.edu/ druginfo.

References

1 Taylor JA, Newman RD. Parental attitudes toward varicella vaccination: the Puget Sound Pediatric Research Network. Arch Pediatr Adolesc Med 2000;154:302-306.
2 O'Neil J. Vital signs: prevention; extra benefit from chickenpox vaccine. New York Times. Health and Fitness. July 4, 2000.

3 Marwick C. Clearing the way for new combination vaccine use [news]. JAMA 2000;283:1280-1281.

4 Neuzil KM, Mellen BG, Wright PF, Mitchel EF Jr, Griffin MR. The effect of influenza on hospitalizations, outpatient visits, and courses of antibiotics in children. N Engl J Med 2000;342:225-231.

5 Izurieta HS, Thompson WW, Kramarz P, et al. Influenza and the rates of hospitalization for respiratory disease among infants and young children. N Engl J Med 2000;342:232-239.

6 McIntosh $\mathrm{K}$, Lieu T. Is it time to give influenza vaccine to healthy infants? [editorial] $N$ Engl J Med 2000;342:275-276.

7 Carman WF, Elder AG, Wallace LA, et al. Effects of influenza vaccination of health-care workers on mortality of elderly people in long-term care: a randomised controlled trial. Lancet 2000;355:9397.

8 Nicholson KG. Should staff in long-stay hospitals for elderly patients be vaccinated against influenza? Lancet 2000;355:83-84.

9 Altman LK. Flu vaccine for the fall is delayed by manufacturing problems. New York Times. National Desk. June 23, 2000.

\section{Netphiles}

\section{Older parents}

Older parents are not only less likely to conceive, but also more likely to have a bad outcome from their pregnancy ( $B M J$ 2000;320:1708-1712).

The human cost of all this is clearly expressed on the Internet. Hannah's Prayer (www.hannah.org/), which has a nondenominational Christian character, links a wide range of resources for women who have lost pregnancies or had stillborn infants. There are also "down the line" health care resources. Searching for information on this well-recognized topic is harder than I thought it would be. The conventional medical portals such as OMNI (www.omni.ac.uk) have lots of general information about pregnancy but little specific on this issue.

The most promising new resource I have encountered is the Open Directory Project (www.dmoz.org/), which, like Yahoo, is a human-edited directory. Unlike Yahoo, it has 25,930 volunteer editors (at the last count) indexing 279,491 categories. Their efforts are made freely available by using an open license and, plainly, their combined might has the potential to overwhelm any commercial venture. It at least produced some relevant hits, a personal account of pregnancy in later years (www. thelaboroflove.com/forum/jan/1.html), and links to consumer resources such as BabyZone (babyzone.com/family.htm).

But the legendary personalization that the Internet is supposed to enable was not much in evidence on BabyZone: zapping advertisements for baby products alongside content aimed at subfertile women and the mothers of recently stillborn infants seems tactless. One way to avoid such offenses would be to accept remaining childless: www.moretolife.co.uk/ offers a support organization for would-be parents who cannot have children.

Douglas Carnall, $B M J$

dcarnall@bmj.com
We welcome

suggestions

for Web sites

to be included

in future

Netphiles 